\title{
Sodium-glucose cotransporter-2 inhibition and acidosis in patients with type 2 diabetes: a review of US FDA data and possible conclusions
}

This article was published in the following Dove Press journal: International Journal of Nephrology and Renovascular Disease 15 June 2017

Number of times this article has been viewed

John A D’Elia'

Alissa R Segal',2

George P Bayliss ${ }^{3}$

Larry A Weinrauch'

'Kidney and Hypertension Section, Joslin Diabetes Center, Harvard Medical School, ${ }^{2}$ Department of Pharmacy Practice, MCPHS University, Boston, MA, ${ }^{3}$ Division of Kidney Diseases and Hypertension, Rhode Island Hospital, Alpert Medical School, Brown University, Providence, RI, USA

Correspondence: Larry A Weinrauch Kidney and Hypertension Section, Joslin Diabetes Center, Harvard Medical School, 52I Mount Auburn Street, Suite 204, Watertown, MA 02472, USA

Email Iweinrauch@hms.harvard.edu
Objective: To evaluate whether adverse event reports to the US Food and Drug Administration on incidents of ketoacidosis from use of sodium glucose cotransport inhibitors (SGLT2 inhibitors) provide insight into ways this new class of drugs is being prescribed with other antihyperglycemic agents; to examine possible mechanisms to explain ketoacidosis.

Design and methods: Reports of adverse events concerned to SGLT2 inhibitors, namely, empagliflozin, dapagliflozin, and canagliflozin were obtained under the Freedom of Information Act for 5 years ending in August 31, 2015. The data were evaluated for incidents of ketoacidosis by looking for keywords such as diabetic ketoacidosis, ketoacidosis, lactic acidosis, acidosis, and metabolic acidosis. Results were tabulated individually for empagliflozin ( $\mathrm{n}=260$ adverse event reports), dapagliflozin ( $\mathrm{n}=520)$, and canagliflozin $(\mathrm{n}=2159)$. Adverse events were categorized according to age, gender, and insulin use.

Results: There were 46, 144, and 450 reports of ketoacidosis concerned with the use of empagliflozin, dapagliflozin, and canagliflozin, respectively. The use of SGLT2 inhibitors was not strictly limited to patients with type 2 diabetes but was cut across categories of insulin use, including a total of 172 cases of SGLT2-related ketoacidosis in individuals above the age of 40 who were not on insulin.

Conclusion: Further studies should focus to detect pleiotropic effects of SGLT2 inhibitors, particularly with other oral antihyperglycemic drugs or insulin. A review of the literature suggests that patients with type 2 diabetes with low C-peptide level may be at increased risk of ketoacidosis, particularly if they are on statins and diuretics due to hypokalemia and impaired release of insulin. More studies are warranted to further clarify these mechanisms.

Keywords: SGLT2 inhibitor, diabetes, ketoacidosis, acidosis, FDA

\section{Introduction}

Sodium-glucose cotransporter (SGLT2) inhibitors are a new class of drugs that work by inhibiting the renal tubular reabsorption of sodium and glucose allowing us to take advantage of glycosuria (glucose excretion) while the patient remains relatively euglycemic. ${ }^{1}$ These inhibitors may also decrease insulin secretion with blood glucose levels that remain close to the normal range due to the blockade of glucose reabsorption at the renal proximal tubule. ${ }^{2}$

Problems have emerged with the use of SGLT2 inhibitors, some of which might have arisen directly from their high level of efficiency. Insulin deficiency due to a decreased secretion or alteration in the dosing of exogenous insulin and lipolysis are two of the predominant hypotheses for the episodes of ketoacidosis that have been 
reported with use of SGLT2 inhibitors. ${ }^{3}$ In addition, risk of dehydration is elevated, as these patients may not increase their fluid intake sufficiently to replace losses due to the use of SGLT2 inhibitors. ${ }^{4}$

Concomitant medications may contribute to the adverse effects of SGLT2 inhibitors. Patients taking diuretics for hypertension or fluid overload, particularly if they don't decrease their diuretic dose, or who are hesitant to increase their fluid intake when initiating an SGLT2, are at higher risk. A recent crossover study on canagliflozin and hydrochlorothiazide found adverse events of mild severity including orthostatic hypotension. ${ }^{5}$ Other drugs commonly used in patients with type 2 diabetes may potentiate problems. The common concomitant use of statins and thiazide diuretics in patients with type 2 diabetes taken to reduce the risk of vascular events may further complicate the problems with SGLT2 inhibitors as they may decrease insulin secretion; however, the use of angiotensin converting enzyme inhibitors may increase insulin secretion.

The US FDA recently mandated a change to the labeling of all SGLT2 inhibitors and expanded pharmacovigilance monitoring for 5 years to further investigate ketoacidosis with these agents. ${ }^{6}$ In a further attempt to understand the scope of the ketoacidosis occurring with SGLT2 inhibitors, this article reviews data reported to the US FDA and discusses evidence regarding the possible etiology.

\section{Methods}

Data received from the US FDA Adverse Event Reporting System obtained under the Freedom of Information Act (detailed reports for events between August 31, 2010 and August 31, 2015) were searched for the following terms: diabetic ketoacidosis, ketoacidosis, lactic acidosis, acidosis, and metabolic acidosis for patients reported to be taking SGLT2 inhibitors. As metabolic acidosis and acidosis were often listed with ketoacidosis on the same report, such cases were considered to represent ketoacidosis. Cases of metabolic or lactic acidosis alone were tabulated separately. Documentation of blood glucose and acid-base data was not available. The requested information that was available included age, gender, and additional associated medications. Results were tabulated individually for empagliflozin ( $n=260$ adverse event reports), dapagliflozin $(n=520)$, and canagliflozin $(n=2159)$ and are presented herein. No effort was made to compare individual drugs with respect to prevalence, incidence, or types of events reported, or to assess dosages or combination drug therapy as the goal was to assess the scope of problems related to acidosis in this class of antidiabetic agents. The analysis was based on reports, not on assessment of individual cases, and may overstate the actual number of cases.

No permission was sought from the institutional review board at the lead author's institution. The data were available to the public under the Freedom of Information Act, de-identified except for age and gender by the US FDA. Reporting to the US FDA is exempt from the provisions of the Health Insurance Portability and Accountability Act (HIPAA). ${ }^{7}$

\section{Results}

\section{For all drugs}

Reports of adverse events reveal that the use of SGLT2 inhibitors was not strictly limited to patients with type 2 diabetes. SGLT2 inhibitor-related events were reported across age groups both with and without insulin.

\section{Empagliflozin}

For empagliflozin (Boehringer Ingelheim International $\mathrm{GmbH}$, Ingelheim, Germany) there were 46 reports of diabetic ketoacidosis ( 27 females, 19 males; ages 17-71 years); insulin use was noted in 17 instances (29 were not on insulin). A total of 16 females and 13 males were not on insulin. Focusing upon patients for whom the recorded ages were over 40 years and were not taking insulin, there were a total of 16 reports ( 8 females, 8 males). An additional 5 reports identified either lactic or metabolic acidosis but not ketoacidosis. A total of 19 cases were reported as off-label use, including 5 cases of off-label use associated with ketoacidosis.

\section{Dapagliflozin}

For dapagliflozin (AstraZeneca AB, Cambridge, UK) there were 144 reports of diabetic ketoacidosis ( 86 females, 45 males, 13 gender unspecified; ages 14-86 years); insulin use was noted in 43 instances (101 were not on insulin). A total of 57 females and 33 males and 11 with gender unspecified were not taking insulin. Focusing upon patients for whom the recorded ages were over 40 years and were not taking insulin, there were a total of 51 reports (36 females, 15 males). An additional 20 reports identified either lactic or metabolic acidosis but not ketoacidosis. A total of 46 cases were reported as off-label use, including 18 cases being associated with ketoacidosis and 2 with ketosis alone.

\section{Canagliflozin}

For canagliflozin (Mitsubishi Tanabe Pharma, Osaka, Japan) there were 450 reports of diabetic ketoacidosis (215 females, 158 males, 67 gender unspecified; ages 16-84 years); insulin 
use was noted in 171 instances (279 were not on insulin). A total of 128 females and 96 males and 55 with gender unspecified were not taking insulin. Focusing upon patients for whom the recorded ages were over 40 years and were not taking insulin, there were a total of 105 reports (59 females, 46 males). An additional 50 reports identified either lactic or metabolic acidosis but not ketoacidosis. A total of 189 cases were reported as off-label uses, including 30 cases of off-label use and ketoacidosis.

\section{Discussion}

As of this writing, the US FDA had listed 172 instances of SGLT2-related ketoacidosis in individuals over the age of 40 years not taking insulin, which can be considered an underestimate of the problem for physicians treating patients with type 2 diabetes.

Analysis of the records provided by the US FDA under the Freedom of Information Act raises several concerns on the basis of both clinical and experimental observations. Data acquired as to gender, age, and associated drugs is often incomplete, rendering analysis of causation at best suspect. The reported data suggests that many physicians are either not aware of current guidelines limiting the use of SGLT2 inhibitors to patients with type 2 diabetes or are using the drugs "off-label", although some investigators are pursuing this group of medications for potential benefit in patients with type 1 diabetes. ${ }^{8}$ Reporting of concomitant drug usage may be inadequate: according to the current data base, fewer than $10 \%$ of the patients taking SGLT2 active drugs were taking statins or aspirin. Analyses of such a database may be adequate to determine if an excess of some events is being reported, but is not sufficiently granular to determine its import.

Reports of efficacy and safety of SGLT2 inhibitor therapy for patients with type 2 diabetes have led the US FDA to approve some combination therapies for glycemic control. ${ }^{9,10}$ For each of these combinations, clinical trials excluded patients with marginal renal function due to either safety concerns or due to the fact that the medication regimen requires adequate renal function to achieve effectiveness. As such, combinations become available as "off-label" use and tend to expand the numbers and types of populations being treated without the safeguards established for a clinical trial. ${ }^{11}$ This may or may not extend the benefits to other populations while potentially increasing the risk of harm. It is only through case reports, series, and such databases or registries such as the US FDA Adverse Event Reporting System that we acquire knowledge as to problems in therapy.
Brief trials of triple antidiabetic therapies are now being published limiting enrollment to patients with an estimated glomerular filtration rate of $>60$. It is known that SGLT2 inhibitors are less effective when renal function is limited due to inadequate filtration of glucose through the glomerulus to the proximal tubule. Consequently, glycemia is not reduced. Extension of SGLT2 inhibitors to patients with renal dysfunction thus has limited benefit, ${ }^{12}$ and if combined with other agents such as metformin, it may potentiate adverse consequences. The combinations of metformin and SGLT2 inhibitor may increase the risk to populations with age-related frailty and/or marginal renal function. No outcome studies have been reported that demonstrate benefit for any of these agents alone or in combination for patients with moderate to severe renal insufficiency (estimated glomerular filtration rate of $<60$ ).

Better understanding of the mechanism of ketoacidosis from SGLT2 inhibitors may help clinicians identify patients at risk as many patients are taking multiple drugs that can affect insulin levels. At the same time, more data is needed on the possible interactions of SGLT2 inhibitors and other drugs that may affect insulin and glucagon levels. According to the literature, in SGLT2 inhibitor-related ketosis, hyperglycemia is corrected through high-grade glycosuria ${ }^{13-15}$ or a low-carbohydrate diet ${ }^{16}$ with subsequent ketosis being due to inadequate secretion of insulin and insulin resistance. SGLT2 inhibitors may diminish the urinary excretion of ketones, eliminating a standard sign of lipolysis in the setting of insulin deficiency with or without glucagon excess. ${ }^{3}$

Umpierrez et al $^{17}$ focused on elucidating differences between insulin-resistant overweight adults who had demonstrated prior ketoacidosis and those who had not. Both groups responded to glucose infusion with equivalent but suboptimal amounts of insulin; however, glucagon stimulation produced a higher insulin output among individuals without prior ketoacidosis than among those with prior ketoacidosis. ${ }^{18}$

Patients with type 2 diabetes are at risk of ketoacidosis if pancreatic insulin secretion is suppressed by hypokalemia induced by the combined effects of SGLT2 inhibitors and diuretics such as hydrochlorothiazide, chlorthalidone, or metolazone. ${ }^{19}$ Insulin secretion may be inhibited by potassium depletion secondary to hydrochlorothiazide or sodium depletion secondary to low-salt diet, independent of aldosterone levels. ${ }^{20}$ There may also be a genetic component with susceptibility to hydrochlorothiazide hypokalemia reported in European and African study populations. ${ }^{21}$ But studies on the benefits of angiotensin converting enzymes have also shown aldosterone-induced reduction in insulin secretion and 
increased insulin resistance. ${ }^{22,23}$ Restoration of normal serum potassium only partially corrected impaired glucose tolerance associated with inhibited insulin secretion, possibly because aging beta cells (as evidenced by amyloid deposition) are capable of secreting both insulin and glucagon. ${ }^{18}$ Glycemic control is sometimes assisted by the use of medications that block the angiotensin-aldosterone system because excess aldosterone has a negative effect on insulin sensitivity and secretion. ${ }^{24-28}$ The effect of such medications on glucose control may not be appreciated until these drugs are discontinued.

Glucagon excess may provide an additional explanation for lipolysis with production of ketones. A study by Bonner et al on alpha cells ${ }^{29}$ has identified an SGLT2 receptor that promotes release of potassium with the entry of calcium, which consequently prevents glucagon secretion. When this receptor has been inhibited, the ensuing entry of potassium with the release of calcium results in the secretion of glucagon. Thus, decreased oxidation of glucose with increased oxidation of fatty acids may occur with SGLT2 inhibitors both by decreasing insulin secretion and by increasing secretion of glucagon. Mechanisms of both inhibition of beta cell function and diminution of insulin sensitivity may involve ATP-dependent potassium channels. ${ }^{30}$ The careful use of lowdose sulfonylurea might be protective by inhibiting secretion of glucagon while stimulating insulin secretion, ${ }^{31}$ but statins may decrease insulin secretion. ${ }^{32}$

The statistical risk of SGLT2-related ketoacidosis in type 2 diabetes is not known as the government institutions - the FDA and the European Medical Agency - are still accumulating the information on rare genetic susceptibilities randomly distributed in diverse populations. Table 1 summarizes an approximation of clinical risk predicted from a crude estimate of beta-cell capacity for insulin secretion by means of

Table I Lists relative risks of undetected ketoacidosis in patients with type 2 diabetes treated with SGLT2 inhibitors. Urine ketones may remain undetected if renal clearance is depressed due to dehydration

\begin{tabular}{ll}
\hline C peptide levels & $\begin{array}{l}\text { Relative } \\
\text { ketoacidosis risk }\end{array}$ \\
\hline C peptide $<$ normal range $(<\mathrm{I} . \mathrm{I} \mathrm{ng} / \mathrm{mL})$ & \\
Treatment includes insulin & $3+$ \\
C-peptide within normal range $(\mathrm{I} . \mathrm{I}-4.2 \mathrm{ng} / \mathrm{mL})$ & \\
Treatment prior to $\mathrm{SGLT2}$ includes insulin & $2+$ \\
$\quad$ Treatment does not include insulin & $\mathrm{I}+$ \\
C-peptide $>$ normal range $(>4.2 \mathrm{ng} / \mathrm{mL})$ & \\
Treatment includes insulin & $\mathrm{I}+$ \\
Treatment does not include insulin & $0+$ \\
\hline
\end{tabular}

Abbreviation: SGLT2, sodium-glucose cotransporter 2 . the C-peptide assay. ${ }^{33}$ An incidence of 1.34 per 1,000 person years has been reported by Danish investigators based on a review of national registries. ${ }^{34}$

In summary, if glucose release in the proximal tubule persists, then ketoacidosis can occur at relatively normal blood glucose concentrations, and if dehydration proceeds to a level of decreased kidney clearance of glucose and ketones, then hyperglycemic ketoacidosis may occur without detection of urine ketones. Clinicians are urged to initiate the use of SGLT2 inhibitors more cautiously in patients with type 2 diabetes who are also taking diuretics and statins. This risk may be accentuated in the elderly. ${ }^{35}$ Thus, in view of recent reports of combined use SGLT2 inhibitors with metformin, ${ }^{36,37}$ patients may experience multiple causes of acidosis, including accumulation of ketones (SGLT2 inhibitors) and lactic acid (metformin) as well as loss of bicarbonate via the gastrointestinal tract with metformin.

\section{Conclusion}

SGLT2 antagonists hold potential of improving cardiovascular and renal outcomes in patients with type 2 diabetes and relatively preserved renal function, particularly when added to drugs that constitute the current standard of care. ${ }^{38}$ But our review of the US FDA data shows that the assessment of the pleiotropic effects of concomitant medications used with SGLT2 inhibitors needs to be further studied to elucidate interactions that may potentiate the adverse effects that have been observed with this new class of medications. Until more information is available to guide them, physicians should use caution in prescribing these new medications to patients with type 2 diabetes who are also taking diuretics and statins, as well as in populations in whom the drugs were not explicitly studied.

\section{Acknowledgment}

This research received no specific grant from any funding agency in the public, commercial, or not-for-profit sectors.

\section{Author contributions}

JAD and LAW contributed to the article conception, data acquisition and analysis, and manuscript drafting and critical revision; ARS contributed to the data acquisition and analysis, manuscript drafting and critical revision; GPB contributed to the data analysis and manuscript revision. All authors approved the final version and agree to be accountable for all aspects of the work. All authors contributed toward data analysis, drafting and critically revising the paper and agree to be accountable for all aspects of the work. 


\section{Disclosure}

The authors report no conflicts of interest in this work.

\section{References}

1. Rieg T, Masuda T, Gerasimova M, et al. Increase in SGLT1-mediated transport explains renal glucose reabsorption during genetic and pharmacological SGLT2 inhibition in euglycemia. Am J Physiol Renal Physiol. 2014;306(2):F188-F193.

2. Ferrannini E, Muscelli E, Frascerra S, et al. Metabolic response to sodium-glucose cotransporter 2 inhibition in type 2 diabetic patients. $J$ Clin Invest. 2014;124(2):499-508.

3. Taylor SI, Blau JE, Rother KI. SGLT2 inhibitors may predispose to ketoacidosis J Clin Endocrinol Metab. 2015;100(8):2849-2852.

4. Bogdanffy MS, Stachlewitz RF, van Tongeren S, et al. Nonclinical safety of the sodium-glucose cotransporter 2 inhibitor empagliflozin. Int J Toxicol. 2014;33(6):436-449.

5. Devineni D, Vaccaro N, Polidori D, Rusch S, Wajs E. Effects of hydrochlorothiazide on the pharmacokinetics, pharmacodynamics, and tolerability of canagliflozin, a sodium glucose co-transporter 2 inhibitor, in healthy participants. Clin Ther. 2014;36(5):698-710.

6. US Food and Drug Administration. FDA drug safety communication: FDA revises labels of SGLT2 inhibitors for diabetes to include warnings about too much acid in the blood and serious urinary tract infections. Available from: https://www.fda.gov/Drugs/DrugSafety/ucm475463. htm. Accessed April 28, 2017.

7. US Food and Drug Administration. HIPAA compliance for reporters to FDA MedWatch. Available from: https://www.fda.gov/safety/medwatch/ howtoreport/ucm085589.htm. Accessed April 28, 2017.

8. Peters AL, Henry RR, Thakkar P, Tong C, Alba M. Diabetic ketoacidosis with canagliflozin, a sodium-glucose cotransporter 2 inhibitor, in patients with type 1 diabetes. Diabetes Care. 2016;39(4):532-538.

9. US Food and Drug Administration. Glyxambi (empagliflozin and linagliptin) tablets. Available from: https://www.accessdata.fda.gov/ drugsatfda_docs/nda/2015/206073Orig1 s000TOC.cfm. Accessed April 28, 2017.

10. US Food and Drug Administration. Xigduo XR (dapagliflozin and metformin HCL) extended-release tablets. Available from: https://www accessdata.fda.gov/drugsatfda_docs/nda/2014/205649Orig1s000TOC. cfm. Accessed April 28, 2017.

11. Bode BW, Garg SK. The emerging role of adjunctive noninsulin antihyperglycemic therapy in the management of type 1 diabetes. Endocr Pract. 2016;22(2):220-230.

12. Ferrannini E, Veltkamp SA, Smulders RA, Kadokura T. Renal glucose handling: impact of chronic kidney disease and sodium-glucose cotransporter 2 inhibition in patients with type 2 diabetes. Diabetes Care. 2013;36(5):1260-1265.

13. Erondu N, Desai M, Ways K, Meininger G. Diabetic ketoacidosis and related events in the canagliflozin type 2 diabetes clinical program. Diabetes Care. 2015;38(9):1680-1686.

14. Ogawa W, Sakaguchi K. Euglycemic diabetic ketoacidosis induced by SGLT2 inhibitors: possible mechanism and contributing factors. J Diabetes Investig. 2016;7(2):135-138.

15. Peters AL, Buschur EO, Buse JB, Cohan P, Diner JC, Hirsch IB. Euglycemic diabetic ketoacidosis: a potential complication of treatment with sodium-glucose cotransporter 2 inhibition. Diabetes Care. 2015;38(9):1687-1693.

16. Hayami T, Kato Y, Kamiya H, et al. Case of ketoacidosis by a sodiumglucose cotransporter 2 inhibitor in a diabetic patient with a lowcarbohydrate diet. J Diabetes Investig. 2015;6(5):587-590.

17. Umpierrez GE, Casals MM, Gebhart SP, Mixon PS, Clark WS, Phillips LS. Diabetic ketoacidosis in obese African-Americans. Diabetes. 1995;44(7):790-795.
18. Spijker HS, Song H, Ellenbroek JH, et al. Loss of $\beta$-cell identity occurs in Type 2 diabetes and is associated with islet amyloid deposits. Diabetes. 2015;64(8):2928-2938.

19. Pfützner A, Klonoff D, Heinemann L, Ejskjaer N, Pickup J. Euglycemic ketosis in patients with type 2 diabetes on SGLT2-inhibitor therapy-an emerging problem and solutions offered by diabetes technology. Endocrine. 2017;56(1):212-216.

20. Luther JM, Byrne LM, Yu C, Wang TJ, Brown NJ. Dietary sodium restriction decreases insulin secretion without affecting insulin sensitivity in humans. J Clin Endocrinol Metab. 2014;99(10):E1895-E1902.

21. Del-Aguila JL, Cooper-DeHoff RM, Chapman AB, et al. Transethnic meta-analysis suggests genetic variation in the HEME pathway influences potassium response in patients treated with hydrochlorothiazide. Pharmacogenomics J. 2015;15(2):153-157.

22. Luther JM, Luo P, Kreger MT, et al. Aldosterone decreases glucosestimulated insulin secretion in vivo in mice and in murine islets. Diabetologia. 2011;54(8):2152-2163.

23. Luther JM. Effects of aldosterone on insulin sensitivity and secretion. Steroids. 2014;91:54-60.

24. Hjelmesaeth J, Jenssen T, Hagen M, Egeland T, Hartmann A. Determinants of insulin secretion after renal transplantation. Metabolism. 2003;52(5):573-578.

25. Huang Z, Jansson L, Sjöholm A. Vasoactive drugs enhance pancreatic islet blood flow, augment insulin secretion and improve glucose tolerance in female rats. Clin Sci (Lond). 2007;112(1):69-76.

26. Luther JM, Brown NJ. The renin-angiotensin-aldosterone system and glucose homeostasis. Trends Pharmacol Sci. 2011;32(12):734-739.

27. Saitoh Y, Hongwei W, Ueno H, Mizuta M, Nakazato M. Candesartan attenuates fatty acid-induced oxidative stress and $\mathrm{NAD}(\mathrm{P}) \mathrm{H}$ oxidase activity in pancreatic beta-cells. Diabetes Res Clin Pract. 2010;90(1):54-59.

28. van der Zijl NJ, Moors CC, Goossens GH, Hermans MM, Blaak EE, Diamant M. Valsartan improves \{beta\}-cell function and insulin sensitivity in subjects with impaired glucose metabolism: a randomized controlled trial. Diabetes Care. 2011;34(4):845-851.

29. Bonner C, Kerr-Conte J, Gmyr V, et al. Inhibition of the glucose transporter SGLT2 with dapagliflozin in pancreatic alpha cells triggers glucagon secretion. Nat Med. 2015;21(5):512-517.

30. Gromada J, Ma X, Høy M, et al. ATP-sensitive K+ channel-dependent regulation of glucagon release and electrical activity by glucose in wildtype and SUR 1 -/- mouse alpha-cells. Diabetes. 2004;53 (Suppl 3): S181-S189.

31. Hattersley AT, Thorens B. Type 2 diabetes, SGLT2 inhibitors, and glucose secretion. N Engl J Med. 2015;373(10):974-976.

32. Zhou J, Li W, Xie Q, et al. Effects of simvastatin on glucose metabolism in mouse MIN6 cells. J Diabetes Res. 2014;2014:376570.

33. Umpierrez GE. Ketosis-prone type 2 diabetes: time to revise the classification of diabetes. Diabetes Care. 2006;29(12):2755-2757.

34. Jensen ML, Persson F, Andersen GS, et al. Incidence of ketoacidosis in the Danish type 2 diabetes population before and after introduction of sodium-glucose cotransporter 2 inhibitors - a nationwide, retrospective cohort study, 1995-2014. Diabetes Care. 2017;40(5):e57-e58.

35. D'Elia JA, Segal AR, Weinrauch LA. Metformin-SGLT2, dehydration and acidosis potential. J Am Geriatr Soc. Epub 2017 Feb 2.

36. Mathieu C, Ranetti AE, Li D, et al. Randomized, double-blind, phase 3 trial of triple therapy with dapagliflozin add-on to saxagliptin plus metformin in type 2 diabetes. Diabetes Care. 2015;38(11):2009-2017.

37. Matthaei S, Catrinoiu D, Celiński A, et al. Randomized, double-blind trial of triple therapy with saxagliptin add-on to dapagliflozin plus metformin in patients with type 2 diabetes. Diabetes Care. 2015;38(11): 2018-2024.

38. Wanner C, Inzucchi SE, Lachin JM, et al; EMPA-REG OUTCOME Investigators. Empagliflozin and progression of kidney disease in Type 2 diabetes. N Engl J Med. 2016;375(4):323-334. 
The International Journal of Nephrology and Renovascular Disease is an international, peer-reviewed open access journal focusing on the pathophysiology of the kidney and vascular supply. Epidemiology, screening, diagnosis, and treatment interventions are covered as well as basic science, biochemical and immunological studies. The manuscript management system is completely online and includes a very quick and fair peer-review system, which is all easy to use. Visit http://www. dovepress.com/testimonials.php to read real quotes from published authors.

Submit your manuscript here: https://www.dovepress.com/international-journal-of-nephrology-and-renovascular-disease-journal 